\title{
The superiority of the pair-comparisons method for scaling visual illusions*
}

\author{
ROGER B. HOWARD, MICHAEL WAGNER, and RICHARD C. MILLS \\ Colgate University, Hamilton, New York 13346
}

\begin{abstract}
Twenty-one Ss scaled the Delboeuf illusion by the methods of pair comparisons, constant stimulus differences, reproduction, and pair reproduction. The method of pair comparisons was clearly superior on all indices of validity, reliability, and efficiency. Pair comparisons forces the $S$ to discriminate between treatment levels and avoids motor responses. Es should consider using pair comparisons or related methods which share these advantages.
\end{abstract}

Most scaling methods can be divided into two groups defined by the set membership of the elements being observed (Coombs, 1964). ${ }^{1}$ Between-sets methods are designed to scale the elements in the experimental set by relating them to the elements in a distinct comparison (C) set. Consider, for example, the problem of scaling a set of visual stimuli. In the method of constant stimulus differences, each experimental stimulus is successively paired with several $C$ stimuli. The $S$ is asked to indicate whether the experimental or $\mathrm{C}$ stimulus has more of the attribute being measured. The value assigned to each experimental stimulus is defined by its point of subjective equality on the scale of $C$ stimuli. In other between-sets methods, such as adjustment and reproduction, the $S$ reconstructs the experimental stimulus either by adjusting a $\mathrm{C}$ stimulus in the former method or frequently by drawing the experimental and $C$ stimuli in the latter. The value assigned to each experimental stimulus is defined by the magnitudes of the reconstructions.

Within-sets methods are designed to scale the elements of the experimental set by relating them to each other. In methods such as pair comparisons, each experimental stimulus is paired with every other one, and the S's task is to judge which member of the pair has more of the attribute being measured. The stimuli are scaled as functions of the frequency that each dominates the others and with no reference to a separate set of C stimuli.

Visual illusions have traditionally been scaled by between-sets methods. Nevertheless, they can be scaled by either group of methods if several experimental stimuli are used. In the Delboeuf illusion (Fig. 1), the inner circle (the test, or $T$, circle) appears larger when surrounded by the outer circle (the induction, or I, circle). Many Es have measured the magnitude of the illusion as a function of the size of the I circle. This can be done by a between-sets method such as constant stimulus differences, in which the subjective size of the

*This research was supported in part by grants to the first author from the Colgate Research Council and from the Sloan Foundation.
$T$ figure is located on the millimeter scale represented by the series of C circles (Ikeda \& Obonai, 1955). Alternatively, the S may make pair comparisons among the T figures (Keats, 1964), which usually results in a scale of normalized (z) scores. ${ }^{2}$

Since illusions may be studied by either group of methods, the selection of a particular method must be based on criteria other than necessity. The two criteria most commonly suggested by psychometricians are validity and reliability; the criterion most commonly used in research is efficiency. A valid method is one

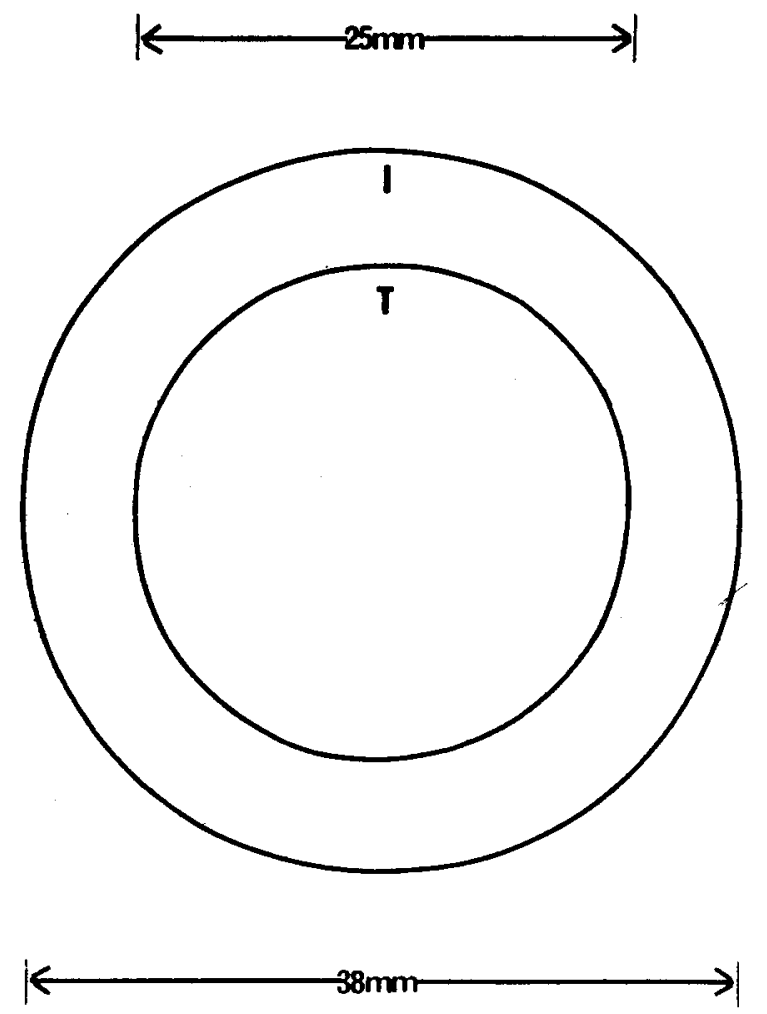

Fig. 1. The basic I-T configuration used in the experiment and its dimensions. 
which scales what the $E$ intends. It depends to a great extent on the E's perception of the task. We would like to suggest that maximum validity means (a) the illusion which is scaled is independent of the specific method and thus is not a scaling artifact: (b) the method has maximum sensitivity for the illusion and the variables which affect it: and (c) the method has minimum sensitivity for extraneous variables which affect the observer or magnify other phenomena but have no direct influence on the illusion. This definition seems compatible with other definitions of validity (Guilford, 1954) and reflects what we feel to be the important characteristics of a valid method. A reliable method is one which gives reproducible data. Although there are several ways of evaluating reliability, we will assume that it is inversely related to the proportion of error variance in the data (Guilford, 1954: Winer, 1971). An efficient method is one which reaches a desired level of stability or statistical significance with the minimum expenditure of resources. Efficiency is sometimes explicitly (Cornsweet, 1962: Feeny, Kaiser, \& Thomas, 1966) and usually implicitly assumed as a criterion. Taken by itself, it certainly does not provide sufficient reason for selecting a method (Dallenbach, 1966). Nevertheless, it serves no purpose to use an inefficient method provided an efficient one exists with comparable validity and reliability.

Although the validity and reliability of most methods are well defined for many perceptual phenomena (Guilford, 1964: Torgerson, 1958; Nunnally, 1967), we have found no systematic analysis of validity, reliability. and efficiency of methods for measuring visual illusions. What data are available suggest that the method of pair comparisons may be superior to the between-sets methods.

Most of the well-known illusions are strong enough to be scaled by most methods, but the methods may not be equally sensitive to very small illusions or differences in treatment effects. Howard and Evans (1971) used the method of reproduction to scale the assimilation illusion produced by a modified version of the Delboeuf figures. The main effects were significant, although the method failed to distinguish among several of the treatment levels. We have since found it impossible to scale the contrast component of the illusion with several versions of the method of reproduction. More recent work in our laboratories suggests that these effects can be scaled by the method of pair comparisons. Keats (1964) obtained similar results with the configuration shown in Fig. 1.

Inefficient methods which require many trials to produce stable data may also lose their sensitivity for some illusions and hence their validity. There is convincing evidence that the magnitude and sign of several illusions change as functions of exposure time (e.g., Judd, 1902, 1905; Judd \& Courten, 1905; Cameron \& Steele, 1905: Lewis, 1908; Köhler \& Fishback. 1950a, b). The method of constant stimulus differences usually requires between 5 and 10 presentations of the stimuli for each measurement at each data point. The method of reproduction may be used with only one presentation of a stimulus configuration, but it often requires many measurements at each point to completely stabilize the data (Guilford, 1954: personal observations). These and related methods would seem to have questionable validity for scaling illusions which are subject to adaptation, whereas our informal observations with the method of pair comparisons and the data cited by Guilford (1954) and Keats (1964) suggest that it produces reliable data with very few trials.

We have also observed that the method of adjustment may lose validity by introducing perceptual errors of movement. We attempted to use the method of adjustment to study the illusion produced by the normal Mueller-Lyer figures and similar figures composed of dots. ${ }^{3}$ Our data indicated that the Ss stopped the adjustment of the dotted figure sooner than they did of the normal figure on both ascending and descending trials $[F(1,47)=14.99 ; p<.001]$. The effect was eliminated by a version of the method of pair comparisons using identical figures.

In the following study. we compare the validity, reliability, and efficiency of the methods of constant stimulus differences, reproduction, pair comparisons. and a fourth method which we call pair reproduction. There may be several sources for the advantages of pair comparisons over the between-sets methods, one of which is direct comparison of the experimental stimuli. If two treatment levels produce almost identical psychological responses, the $S$ is more likely to detect the small differences between the stimuli and make consistent responses to them if the stimuli are presented on the same trial rather than on different trials. In the method of pair reproduction. we extend this possible advantage to the method of reproduction and ask the $S$ to reproduce all pairs of experimental stimuli. If the method of pair comparisons is superior because of the direct comparisons of the $T$ figures, then pair reproduction should produce comparable data.

\section{METHOD}

\section{Subjects}

The Ss were 16 male and 5 female undergraduates from Colgate University. They were each paid \$2 for their participation in the experiment.

\section{Design and Procedure}

The independent variable was the diameter of the I circle in the Delboeuf configuration shown in Fig. 1. The diameters used in the study were $25.0,31.5 .38 .5,44.5$, and $51.0 \mathrm{~mm}$. The diameters of the eight $C$ figures used in the method of constant stimulus differences ranged from 23.0 to $30.0 \mathrm{~mm}$ in $1.0-\mathrm{mm}$ steps. The diameters of the $\mathrm{C}$ figure in the method of reproduction and the $T$ figure in 11 conditions were $25.0 \mathrm{~mm}$.

Each I-T configuration (i.e.. combination of the $T$ figure with one of the I figures and each C figure was drawn in black ink on 
a separate $15.2 \times 22.8 \mathrm{~cm}$ white card. The pairs of cards were shown at a distance of $2.0 \mathrm{~m}$ with $22.8 \mathrm{~cm}$ between the centers of the circles being compared. The illuminance of the cards and the background were 10.0 and $5.5 \mathrm{fc}$, respectively. All viewing was monocular with the S's preferred eye.

The 160 trials in the method of constant stimulus differences were divided into 20 blocks of eight trials, each defined by pairing the eight $C$ figures with one of the five I-T configurations. The left-right positions of the $\mathrm{C}$ and I-T configurations were the same for all trials within a block. There were four blocks for each of the five I-T configurations, two with the $\mathrm{C}$ figure on the left and two with it on the right. The order of trials within a block and the order of the blocks were both randomized for each $\mathrm{S}$. His task was to indicate whether the $\mathrm{T}$ or $\mathrm{C}$ circle was larger.

In the method of reproduction, each of the five I-T configurations was paired with the $25.0-\mathrm{mm} C$ figure four times for a total of 20 trials, which were presented in a different random order for each $S$. The $S$ was asked to draw two vertical lines which had the same lengths as the vertical diameters of the $T$ and $C$ circles. Each drawing was made on a separate $13.0 \mathrm{x}$ $10.0 \mathrm{~cm}$ piece of white paper.

The method of pair comparisons and the method of pair reproduction were combined into a single set of trials. Each of the five I-T configurations was paired once with each of the other four configurations for a total of 20 trials, which were presented in a different random order for each S. On each trial, the $S$ was asked to indicate which of the two $T$ circles was larger and then to draw their vertical diameters in the same manner described above for the method of reproduction. The pair-comparisons data were the frequencies with which each stimulus was judged larger. The pair-reproduction data were the lengths of the drawn lines. The order of the three sets of trials was counterbalanced within groups of three Ss.

\section{DATA ANALYSIS}

It is desirable, for mathematical reasons, to locate the stimuli on an interval or ratio scale. The scaling methods often produce raw data which have nominal or ordinal properties and which must therefore be transformed by a scaling model. In the following analysis, we construct an interval scale for each set of data based on the most common scaling model.

In the method of pair reproduction, the $S$ drew the vertical diameters of the $T$ circles. The data are thus in millimeters and have ratio-scale properties. It seems reasonable to assume that the magnitude of the illusion is best represented by the mean length of the reproduction. Figure 2 shows mean length plotted as a function of the diameter of the I circle.

The raw data from the method of reproduction also have ratio properties, but the scale resulting from the application of the traditional model is not the scale found for pair reproduction. Although the reproduction of each $\mathrm{T}$ figure could be measured directly and averaged as in the former method, the conventional procedure is to take the difference between the reproductions of the $T\left(E_{t}\right)$ and $C\left(E_{c}\right)$ figures and convert them to percentage of illusion scores by the formula: percentage of illusion $=\left[\left(E_{t}-E_{c}\right) / E_{c}\right] \times 100$. Figure 2 shows the mean percentage of illusion scores for each I-T configuration.

In the method of constant stimulus differences. the

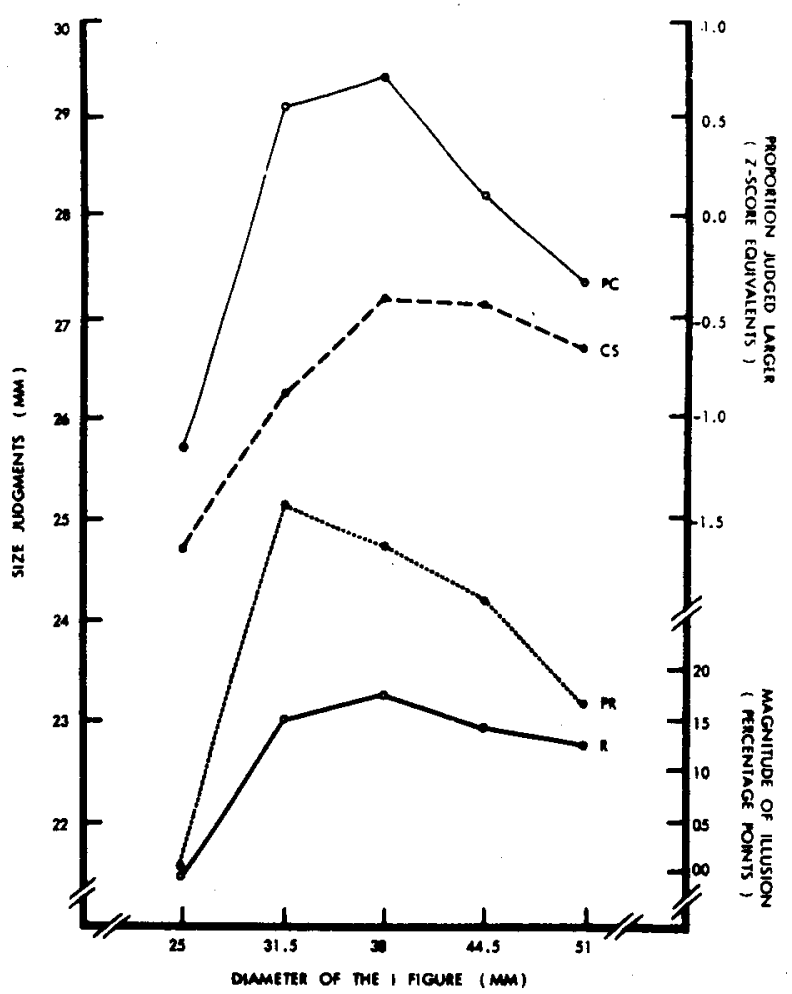

Fig. 2. The average values of the scaled data as functions of the diameter of the 1 figure. The data from the methods of constant stimulus differences (CS) and pair reproduction (PR) are scaled in millimeter units (the left ordinate of the figure). The data from the method of pair comparisons (PC) are scaled in $z$-score units (the upper right ordinate) and the data from the method of reproduction are scaled as percentages of illusion (the lower left ordinate).

raw data are the frequencies at which the $S$ judges each of the $\mathrm{C}$ stimuli to be larger than the $\mathrm{T}$ stimulus. In order to construct an interval scale, the model assumes that the frequencies are normally distributed over the $\mathrm{C}$ stimuli. The problem of scaling then reduces to fitting an ogive to the distribution relating "larger" responses to the magnitude of the $\mathrm{C}$ figure. Sample data were analyzed by each of the seven methods described by Guilford (1954), and the complete data were analyzed by the summation method and the least-squares procedures with and without the Mueller-Urban weights. All methods gave very similar results. The product-moment correlations between the three methods used on the complete data were all above 0.98 . Figure 2 shows the results from the least-squares solution using the Mueller.Urban weights, as this solution best fits the scaling model.

It is possible to compute a chi-square test for goodness of fit between the scaling model and the data. The formula is given by Guilford (1954). It yields chi squares of $3.77,8.40,4.59 .7 .63$, and 8.34 with $5 \mathrm{df}$ for each of the five I-T configurations ordered from 25.0 to $51.0 \mathrm{~mm}$. None is significant $(p>.05)$. Thus. the 
model fits the data reasonably well.

The model underlying the method of pair comparisons is based on the assumption that the magnitude of the separation between the psychological values of two stimuli may be determined by the frequency at which each is judged to be larger than the other. In order to define an interval scale for the data, it is necessary to make several additional assumptions. The simplest solution to the scaling problem is Thurstone's Case $\mathrm{V}$, which is based on the assumption that the judgments for each stimulus are normally distributed as independent random variables with equal variances. The value of the stimulus on the scale is computed by taking the proportion of trials on which the stimulus is judged to be larger than the others and converting that to a $Z$ score. A less restrictive test is provided by Thurstone's Case III. in which the variances are not assumed to be equal. In this case, the $E$ must first estimate the variances and then compute the $z$ scores (Burros. 1951).

As with the method of constant stimulus differences. it is possible to test the goodness of fit between each case of the model and the data. Mosteller (1951) has presented the rationale and one set of formulas for these computations. Gulliksen and Tukey (1956) provide an even more general analysis of variance procedure, which not only yields an angular chi square similar to the one suggested by Mosteller, but also gives several reliability coefficients. The data from the pair-comparison method were analyzed under the assumptions of both Case III and Case V. The chi square for Case V was not significant $\left[\chi^{2}(6)=9.98, p>.10\right]$, which indicates that the model fits the data reasonably well. The solution for Case III yields a much smaller value $\left[\chi^{2}(2)=2.86\right.$, $p>.10]$, which is an improvement despite the lower degrees of freedom. Figure 2 thus shows the mean $z$ scores for each stimulus configuration computed by Thurstone's Case III solution.

\section{RESULTS AND DISCUSSION}

The definitions of validity, reliability, and efficiency assumed earlier may now be operationalized in a straightforward manner. A valid measure should show the presence of an illusion and treatment effects. given reasonable evidence that they exist. The Delboeuf illusion has been studied extensively over the range of treatment levels used in this experiment and has always produced an increase in the size of the $T$ figure which was an inverted-U function of the I-circle diameter (i.e., Ikeda \& Obonai. 1955; Suto, 1961: Keats, 1964: Piaget, 1969). The consistency of this result suggests that a valid method should produce similar data. Figure 2 shows that the data from all four methods are inverted-U functions of the diameter of the I figure.

A valid method should also be very sensitive to treatment effects. Validity in this sense depends on reliability. A method is sensitive if it can distinguish among very small treatment effects. That is. it is sensitive if the error variance in the data is small relative to the treatment variance. This definition is clearly related to the definition of reliability suggested by Guilford (1954) and Winer (1971) and assumed above Thus. a method must be reliable if it is to be valid. and measures of reliability provide information about validity as well.

We have computed five reliability coefficients. all of which show the high reliability of the method of pair comparisons. Winer (1971) suggests two reliability coefficients, $r_{k}$ and $r_{1}$, based on the analysis of variance. When transformed to match our data, $r_{k}$ measures the reliability of using 21 Ss to judge the five stimuli. $r_{1}$ measures the reliability of using a single average $S$. The data were first submitted to $F_{\max }$ tests (Winer. 1971). which showed that there were no significant differences in the variances at each treatment level within each method $(p>.05)$. Consequently, the $F$ ratios were computed from the results of one-way analyses of variance, with the treatments mean squares divided by the Treatments by Ss interaction mean squares. The $r_{k} s$ were $0.99,0.98,0.95$, and 0.94 for the methods of pair comparisons, constant stimulus differences. pair reproduction. and reproduction. respectively. The $\mathrm{r}_{1} \mathrm{~s}$ were $0.79,0.67,0.50$. and 0.41 for the methods. in the same order. Thus, pair comparisons is the most reliable of the four methods.

Although these analyses of variance allow comparisons among the different methods, better measures of reliability for the method of pair comparisons alone may be derived from the variance component analyses obtained in conjunction with the angular chi-square test described above (Gulliksen \& Tukey, 1956). $r_{s}$ and $r_{s s}$ are coefficients of linear consistency and estimates of the reliability of the variance components which fit the linear scaling model. Both represent the reliability of using 21 Ss to judge five stimuli. $I_{s}$ is based on the assumption that the sums of squares for the linear, nonlinear, and error terms need not equal the total sum of squares. Its value is 0.83 for the Case V solution and 0.94 for the Case III solution. $r_{s s}$ is based on the assumption that the components equal the total sum of squares. Its value is 0.93 for the Case V solution and 0.94 for the Case III solution. A third coefficient, $r_{b}$, is theoretically less conservative. because it treats the systematic deviation from the linear scale as treatment rather than error variance. It yields the same value, 0.94. for both cases. Thus, all of the coefficients computed for the pair-comparisons data indicate that the method is highly reliable and that when the same coefficient is computed for all four methods. pair comparisons is shown to be the most reliable.

By the definition given above, the efficiency of a method is a partial function of the number of Ss $(\mathrm{N})$ needed to reach a certain level of significance. Most studies report the $F$ ratio for the entire set of Ss. In analyzing efficiency. it is more important to know the minimum $X$ needed to exceed a given significance level. 
Thus, the maximum information about the efficiency of a method may be obtained by studying the $F$ ratio as a function of $\mathrm{N}$.

The counterbalanced design required three Ss for each replication. The data from each method were submitted to $F_{\max }$ tests with $N=3,6,9, \cdots, 21$. None of the 28 tests was significant $(p>.05)$. Consequently, the data were analyzed by the same one-way analyses of variance used to compute reliability coefficients. The results are shown in Fig. 3.

It is obvious that the method of pair comparisons is superior to the other three methods when the $F$ ratio is used as a measure of efficiency. At no point is the $F$ ratio for pair comparisons lower than the ratios for the other methods. Further, the probability of a Type I error computed from the pair-comparisons data is less than 0.025 with three Ss and 0.001 with six, whereas the probability of a Type I error computed from the other methods is greater than 0.05 with three Ss and requires nine or more Ss before it is less than 0.001 .

It is also interesting to note that the $F$ ratio is a linear function of $\mathrm{N}$ for the pair-comparisons and pair-reproduction data, whereas it is not even a monotonically increasing function for constant stimulus differences and reproduction. The correlation of $F$ ratio with $\mathrm{N}$ is $0.999,0.984,0.948$, and 0.840 for each method, in the order just given. The $\mathrm{F}$ ratio is structured so that the treatments mean squares is a function of $\mathrm{N}$, whereas the error mean squares is not. Thus, the linear function indicates that each group of three Ss contributes almost identical amounts of variance to the pool for the method of pair comparisons, whereas the amount varies for the other methods. This might be considered a measure of the reliability of the efficiency; any three of our Ss would produce significance at the 0.025 level with the method of pair comparisons, any six at the 0.001 level, and so on.

The time and trials required by each method, taken in conjunction with the $F$ ratios also indicate the superior efficiency of the pair-comparisons method. The pair comparisons required 20 trials and an average of $5.5^{4} \mathrm{~min}$ of stimulus exposure under the conditions of this experiment. The method of constant stimulus differences reached a consistently lower $F$ ratio with 160 trials and an average of $24.7 \mathrm{~min}$ of exposure. The reproduction and pair-reproduction methods required 20 trials, but achieved a much lower $F$ ratio in 8.7 and $8.2 \mathrm{~min}$. respectively. A one-way analysis of variance over the times was significant $[F(3,20)=8.23, p<.05]$. Scheffé's post hoc comparisons (Hays, 1963) show that the times for all four methods are significantly different from each other $(p<.05)$, with the exception of reproduction and pair reproduction. Thus, the method of pair comparisons is more valid. reliable, and efficient than the other three methods.

\section{GENERAL DISCUSSION}

It is possible to rank order the methods as measures of

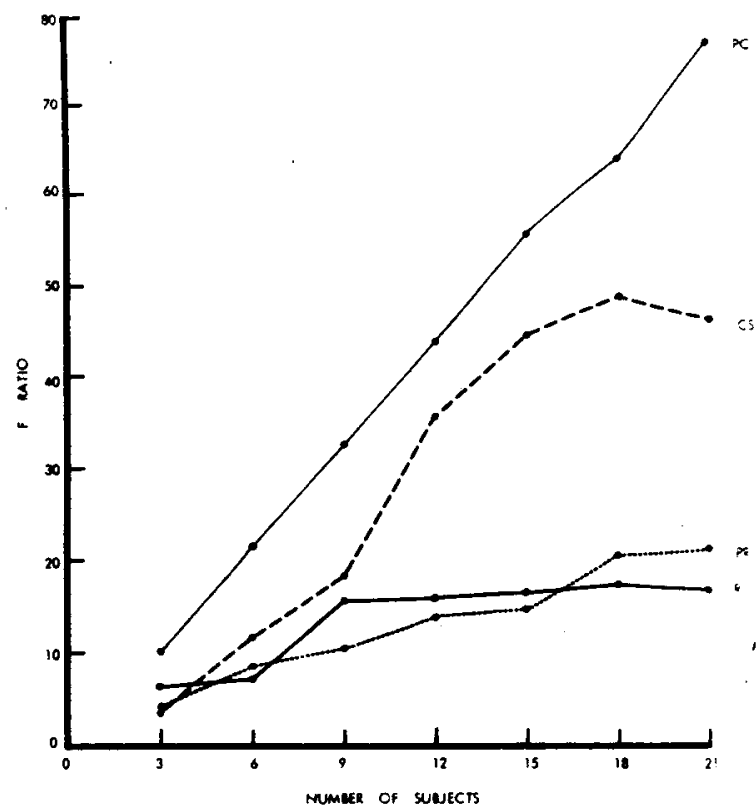

Fig. 3. The magnitudes of the $F$ ratios as functions of the number of Ss. The abbreviations are the same as in Fig. 2.

the Delboeuf illusion on the basis of the statistical analyses of the data. Pair comparisons is clearly the preferred method. Its reliability coefficients, $F$ ratios, linearity of $\mathrm{F}$ ratio as a function of $\mathrm{N}$, and other measures of efficiency (exposure time and trials) are all superior to the corresponding statistics for the other methods. The order of the other three methods depends on the weights assigned to each statistic. Constant stimulus differences is second in reliability coefficients and $F$ ratios, but third in linearity of $F$ ratio as a function of $\mathrm{N}$ and fourth on the other efficiency measures. Pair reproduction is third in reliablity, third or fourth on $F$ ratio (depending on whether the final or average $F$ ratio is used as the statistic), and second on the latter two statistics. We would prefer constant stimulus differences over pair reproduction because of the greater importance of the reliability coefficients and the magnitude of the $F$ ratios in our conceptualization of a good method. Reproduction is last in relíability, third or fourth in $F$ ratio, last in linearity of the $F$ ratio as a function of $\mathrm{N}$, and third on the other efficiency measures. We would rank order it last for the same reasons.

The consistent superiority of the method of pair comparisons probably results from at least two factors. First, the $S$ is forced to compare the experimental stimuli with each other and thus to directly discriminate among them. In the methods of constant stimulus differences and reproduction, he relates each stimulus to a different set of $\mathrm{C}$ stimuli. and does not have to discriminate one stimulus from the other. Thus. the pair comparisons may amplify small differences between the treatment levels by forcing the $S$ to choose between stimuli which are difficult to discriminate from each other. 
The second factor is the susceptibility of the method to extraneous variables. If direct comparison were the only factor. then the method of pair comparisons and pair reproduction would produce comparable data. Although both share the linear relationship between $F$ ratio and $\mathrm{N}$, the other statistics are considerably lower for the latter method. Since the data for both were collected on the same trials, the difference must depend on the nature of the responses that the $S$ was required to make. Methods of reproduction have been criticized in other contexts for introducing motor errors (Guilford, 1954: Fullerton \& Cattell, 1892). It seems likely that the S's reproductions contained motor-error components which reduced the $F$ ratio and the reliabiability coefficients.

In general, our data suggest that the method of pair comparisons may be superior to between-sets methods for measuring visual illusions. Whether it is superior to other within-sets methods is uncertain. It is possible to order many of these methods from pair comparisons, in which $S$ compares two stimuli on each trial, to rank ordering, in which he compares all of the stimuli on a single trial. If comparing two stimuli allows the $S$ to make valid and reliable discriminations among stimuli, then comparing all stimuli simultaneously may further strengthen the data. The method of rank ordering has the additional advantage of being extremely efficient. Each $S$ has one trial with all of the stimuli. Although the length of that trial will vary with the number of experimental stimuli (k), it is unlikely to take as long as the $k(k-1)$ comparisons used in the completely balanced method of pair comparisons. We are currently investigating the relative validity, reliability and efficiency of several within-sets measures, including pair comparisons and rank-ordering.

\section{REFERENCES}

Burros, R. H. The application of the method of paired comparisons to the study of reaction potentials. Psychological Review, 1951, 58, 60-66.

Cameron, E. H., \& Steele, W. M. The Poggendorf illusion. Psychological Review Monograph Supplement, 1905, 7, 83-111.

Coombs, C. H. A theory of data. New York: Wiley, 1964.

Cornsweet, T. N. The staircase-method in psychophysics. American Journal of Psychology, 1962, 75, 485-491.

Dallenbach, K. M. The staircase-method critically examined. American Journal of Psychology, 1966, 79, 654-656.

Feeny, S., Kaiser, P. K., \& Thomas, J. P. An analysis of data gathered by the staircase-method. American Journal of Psychology, 1966, 79. 652-654.

Fullerton, G. S., \& Cattell. J. McK. On the perception of small differences. Publications of the University of Pennsylvania, Philosophical Series, No 2. 1892.

Guilford, J. P. Psychometric methods. (2nd ed.) New York: McGraw-Hill, 1954.

Gulliksen, H., \& Tukey, J. W. Reliability for the law of comparative judgment. Psychometrika. 1958, 23, 95-110.

Hays. W. C. Statistics for psychologists. New York: Holt. Rinehart \& Winston. 1963.

Howard, R. B., \& Evans, G. W. Some evidence for the size-modification theories of illusions. Psychonomic Science, 1971. 23. 129-131
Ikeda, H.. \& Obonai. T. The studies in figural after-effects: IV. The contrast-confluence illusion of concentric circles and the figural after-effect. Japanese Psychological Research, 1955, 2. 17-23.

Judd, C. H. Practice and its effects on the perception of illusions. Psychological Review, 1902, 9, 27-39.

Judd, C. H. The Muller-Lyer illusion. Psychological Review Monograph Supplement, 1903, 7, 55-81.

Judd, C. H., \& Courten, H. C. The Zöllner illusion. Psychological Review Monograph Supplement, 1905, 7, 112-139.

Keats, J. A. An application of the method of paired comparisons to the study of the Delboeuf illusion. Australian Journal of Psychology, 1964, 16, 169-174.

Köhler, W., \& Fishback, J. The destruction of the Müller-Lyer illusion in repeated trials: I. An examination of two theories. Journal of Experimental Psychology. 1950a, 40, 267-281.

Köhler, W.. \& Fishback, J. The destruction of the Müller-Lyer illusion in repeated trials: Il. Satiation patterns and memory traces. Journal of Experimental Psychology, 1950b, 40, 398-410.

Lewis, E. O. The effect of practice on the Müller-Lyer illusion. British Journal of Psychology, 1908, 2, 294-306.

Mosteller, F. Remarks on the method of paired comparisons: III. A test of significance for paired comparisons when equal standard deviations and equal correlations are assumed. Psychometrika, 1951, 16, 207-218.

Nunnally, J. C. Psichometric theory. New York: McGraw-Hill, 1967.

Piaget, J. The mechanisms of perception. New York: Basic Books, 1969.

Suto, Y. Comparative study of assimilation-contrast illusion, figural after-effect and time error for extents of circle and line stimuli. Japanese Psychological Research, 1961, 3, 1-16.

Torgerson, W. S. Theory and methods of scaling. New York: Wiley, 1958.

Winer, B. J. Statistical principles in experimental design. (2nd ed.) New York: McGraw-Hill. 1971.

\section{NOTES}

1. Coombs (1964) presents three dichotomous variables which may be used to categorize data and scaling methods. He assigns names to the four major quadrants defined by two of the variables, but does not formally name the levels of the variables themselves. We have selected one variable for study here and assigned the two levels the names between-and within-sets comparisons. The terms "comparisons" is usually omitted to avoid confusion with the method of pair comparisons.

2. It is possible to adjoin the $z$ scale derived by pair comparisons to a physical scale if two or more $C$ stimuli of different sizes are included within the series of experimental stimuli. If the $\mathrm{C}$ stimuli are assumed to be some function of the physical scale, then each experimental stimulus may be assigned a physical value by interpolation or extrapolation from the $z$ scale to the physical scale. The use of this procedure is probably misleading. Stimuli in a series are perceived relative to each other and the psychological values of the $C$ stimuli are rarely isomorphic with values on a physical scale. The values assigned to a stimulus, whether they are in millimeters or $z$ scores, are perhaps best considered to be in psychological and not physical units.

3. Howard, R. B., \& Wagner, M. The possible role of extrastriatal visual systems in the Mueller-Lyer illusion. In preparation.

4. The exposure times for the method of pair comparisons were obtained from a similar study in which 17 Ss chose the larger $\mathrm{T}$ circle without drawing their diameters.

(Received for publication June 30. 1972: revision received January 30.1973. ) 